\title{
Hallazgos clínicos y de laboratorio que sugieren tempranamente el síndrome de HELLP en pacientes con preeclampsia severa
}

\author{
Victoria García V. ${ }^{1}$, Luis Guillermo Echavarría ${ }^{2}{ }^{2}$, Ricardo Ardila $C^{3}{ }^{3}$, Jorge Gutiérrez $M .^{4}$ \\ ${ }^{1}$ Departamento de Ginecología y Obstetricia-Medicina Crítica y Cuidado Intensivo, ${ }^{2}$ Departamento de Ginecología y \\ Obstetricia, ${ }^{3}$ Departamento de Medicina Interna, Epidemiología, ${ }^{4}$ Departamento de Medicina Materno Fetal. Clínica \\ Universitaria Bolivariana. Medellín, Colombia.
}

\section{RESUMEN}

Objetivo: Determinar la utilidad de las pruebas de laboratorio y clínicas que se alteran de forma precoz para el diagnóstico del síndrome de HELLP en pacientes con preeclampsia severa. Métodos: Estudio observacional retrospectivo, de utilidad de prueba diagnóstica, basado en una cohorte clínica que incluyó mujeres embarazadas con diagnóstico de preeclampsia severa ingresadas entre el 4 de mayo del 2005 al 7 mayo del 2008 en la Clínica Universitaria Bolivariana (CUB). Se realizó comparación entre los dos grupos (HELLP y no HELLP) en las variables estudiadas. Se calculo la sensibilidad, la especificidad, los valores predictivos y las LR (+ y -), y sus IC al $95 \%$, comparando cada una de las variables clínicas, con la prueba oro: diagnóstico final de HELLP. Se realizaron curvas ROC para las pruebas cuantitativas en la hospitalización para identificar cual es el parámetro de laboratorio que mejor determina el desarrollo del síndrome de HELLP. Resultados: El dolor en epigastrio fue el factor clínico predictor más importante en los pacientes con pre eclampsia severa que desarrollaron HELLP con un valor de $p<0,0001$; de las pruebas de laboratorio realizadas al ingreso, las transaminasas fueron significativamente mayores en las pacientes con preclampsia severa que desarrollaron síndrome de HELLP. Conclusión: En las pacientes con diagnóstico de preeclampsia severa la presencia de epigastralgia y/o transaminasas elevadas son hallazgos que sugieren tempranamente el desarrollo de síndrome de HELLP.

\section{PALABRAS CLAVE: Preeclampsia, síndrome de HELLP, factores predictores}

\section{SUMMARY}

Objectives: The aim of this study was to determine the usefulness of precociously altered clinical and laboratory tests for the diagnosis of HELLP in patients with severe preeclampsia. Methods: An observational study was performed, utility of diagnostic tests, based on a clinical cohort which included pregnant women diagnosed with severe preeclampsia admitted between May 4, 2005 and May 7, 2008 in the Clinica Universitaria Bolivariana was conducted. Comparison was made between the two groups (HELLP and non HELLP) on the assessed variables. Sensitivity, specificity, predictive values and the LR (+ and -) and their $95 \% \mathrm{Cl}$ were calculated, comparing each of the clinical variables with the golden standard test: final diagnosis of HELLP. ROC curves were performed for the laboratory quantitative tests during hospitalization to identify which is the laboratory parameter that best determines the development of HELLP syndrome. Results: Epigastrium pain was the most important predictive factor in clinical patients with severe preeclampsia who developed HELLP with a value of $p<0.0001$. The values of laboratory tests and transaminases conducted at admission 
were significantly higher in patients with severe preeclampsia who developed HELLP syndrome. Conclusion: This study found that in patients diagnosed with severe preeclampsia, the presence of epigastric pain and / or transaminases are findings suggest the early development of HELLP syndrome, for that reason changes of liver tests and the presence of epigastric pain should be actively tracked in this group of patients.

\section{KEY WORDS: Preeclampsia, HELLP syndrome, predictors}

\section{INTRODUCCIÓN}

El síndrome de HELLP (Hemolysis, Elevated Liver enzymes, Low Platelets) es una complicación de la preeclampsia severa (PES) que puede desarrollarse en el $10 \%$ de esos casos (1).

La presentación clínica del síndrome de HELLP es variada, inclusive la hipertensión puede estar ausente en un $20 \%$ de las pacientes (2), por lo cual se requiere un control de laboratorio y clínico frecuente en las pacientes con PES dada la alta morbilidad que este síndrome presenta, como coagulación intravascular diseminada (15\%), abruptio de placenta $(9 \%)$, edema agudo de pulmón ( $8 \%)$, falla renal aguda (3\%), falla hepática $(1 \%)$, y síndrome de dificultad respiratoria del adulto, sepsis y accidente cerebro-vascular en el $1 \%$ de las pacientes (3). Igualmente, este síndrome aumenta la mortalidad y morbilidad perinatal con un mortalidad de 74 a 200/1000 nacidos vivos (4). Esta alta mortalidad perinatal es principalmente experimentada en las edades gestacionales muy tempranas (menos de 28 semanas), asociada a restricción del crecimiento intrauterino (RCIU), abruptio de placenta y prematurez $(5,6)$.

El objetivo del estudio es determinar cuáles pruebas de laboratorio y síntomas clínicos se alteran de forma precoz para predecir tempranamente la aparición del síndrome de HELLP en pacientes con PES.

\section{PACIENTES Y MÉTODOS}

Se realizó un estudio observacional, retrospectivo, de una cohorte de pacientes con diagnóstico de PES de la ciudad de Medellín, admitidas en la Clínica Universitaria Bolivariana, para evaluar la utilidad de varias pruebas diagnósticas en la predicción temprana del síndrome HELLP. Fueron incluidas en el estudio mujeres con diagnóstico de PES entre Mayo de 2005 y Mayo de 2008. Se excluyeron pacientes con PES que adicionalmente tuvieran afección renal crónica y hepática, o hematológica que produzca trombocitopenia y/o anemia hemolítica ya conocida, y pacientes cuya historia clínica no tuviera todas las variables a estudio. Esta investigación fue aprobada por el Comité de Investigación y de Ética de la Clínica Universitaria Bolivariana (CUB). El análisis de información se realizó utilizando Epiinfo versión 7 y SPSS versión 17.
Se recolectaron en total 192 pacientes con PES en las que se registró variables clínicas y demográficas, como semanas de gestación, síntomas clínicos de cefalea, epigastralgia y náuseas, a su ingreso al servicio de urgencias obstétricas de la CUB y luego cada 6 horas, al igual que parámetros de laboratorio de LDH, AST, ALT y plaquetas solicitadas al ingreso y durante la evolución intrahospitalaria. El síndrome de HELLP fue determinado por la presencia de los siguientes criterios: hemólisis (caracterizada por una $\mathrm{LDH}>600 \mathrm{U} / \mathrm{L}$ ), elevación de las enzimas hepáticas (AST $>70 \mathrm{U} / \mathrm{L}$ ) y trombocitopenia (PLT $<150.000 / \mathrm{mm} 3)$, clasificación propuesta por Martín y cols $(7,8)$.

Se realizó un análisis descriptivo de las variables del estudio, y un análisis multivariado donde se calculó la sensibilidad, la especificidad, el valor predictivo positivo, el valor predictivo negativo, la LR+ y LR-, y sus intervalos de confianza al 95\%, comparando cada una de las variables clínicas y de laboratorio, con la "prueba de oro": diagnóstico final de HELLP. Adicionalmente se realizaron curvas ROC para las pruebas cuantitativas al inicio de la hospitalización para poder identificar cuál es el parámetro de laboratorio que predice el desarrollo de síndrome de HELLP con mayor probabilidad, y el parámetro clínico con mayor sensibilidad y especificidad para el síndrome de HELLP.

\section{RESULTADOS}

De las 192 pacientes ingresadas con diagnóstico de PES, 64 pacientes desarrollaron síndrome de HELLP y 128 permanecieron con diagnóstico de PES sin progresión a HELLP (Tabla I).

Hubo diferencias en la edad gestacional de ingreso, siendo significativamente menor en las que desarrollaron HELLP (31 vs. 36 semanas; $p<0,0001$ ). Al comparar las características clínicas, se observó mayor incidencia de dolor epigástrico en las pacientes que desarrollaron síndrome HELLP que en las mujeres que permanecieron con el diagnóstico de PES $(71,9 \%$ vs. $3,9 \% ; p<0,0001)$, mientras que la presencia de cefalea durante la evolución se observó más en las pacientes que permanecieron con diagnóstico de PES que en las pacientes que desarrollaron síndrome de HELLP ( $88,3 \%$ vs. $65 \% ; p<0,0001)$. La presencia de náuseas y vómitos no mostró diferencia significativa en ambos grupos $(p=0,318)$. 
Tabla I

CARACTERÍSTICAS CLÍNICAS Y DE LABORATORIO DE LAS PACIENTES ESTUDIADAS

\begin{tabular}{lcccc}
\hline Variable & PES con HELLP & PES sin HELLP & Total & Valor $p$ \\
\hline Edad gestacional (semanas) & $31,9 \pm 3,9$ & $36,1 \pm 2,5$ & $34,7 \pm 3,6$ & $<0,0001^{\star *}$ \\
Cefalea al ingreso & $42(65,5 \%)$ & $113(88,3 \%)$ & $155(80,7 \%)$ & $<0,0001^{\star}$ \\
Epigastralgia al ingreso & $46(71,9 \%)$ & $5(3,9 \%)$ & $51(26,6 \%)$ & $<0,0001^{\star}$ \\
Náuseas o vómitos & $12(18,8 \%)$ & $17(13,3 \%)$ & $29(15,1 \%)$ & $0,318^{\star}$ \\
DHL ingreso & $686,9 \pm 265,1$ & $547,8 \pm 150,3$ & $594,2 \pm 206,2$ & $<0,0001^{\star *}$ \\
AST ingreso & $50,4 \pm 12,1$ & $23,2 \pm 8,7$ & $32,2 \pm 71,2$ & $<0,0001^{\star *}$ \\
ALT ingreso & $46,1 \pm 10,9$ & $21,75 \pm 7,7$ & $30,1 \pm 64,3$ & $<0,0001^{\star *}$ \\
Plaquetas ingreso (x 1000/ul) & $204,6 \pm 62,9$ & $239,6 \pm 59,4$ & $227,9 \pm 62,7$ & $<0,0001^{\star *}$ \\
\hline
\end{tabular}

Las variables cuantitativas están descritas como Media \pm DE. PES: Preeclampsia severa. *Chi-cuadrado de Pearson, sin corregir. ${ }^{* *}$ Prueba de Mann Withney.

Al comparar los parámetros de laboratorio al ingreso y durante la evolución de las pacientes con diagnóstico de PES se apreció que los valores de la transaminasas oxalacética/AST y transaminasa pirúvica/ALT fueron significativamente mayores en las pacientes que desarrollaron síndrome de HELLP (AST: $50,4 \pm 12,1$ vs. $23 \pm 8 \mathrm{U} / \mathrm{L}$ y ALT: 46 $\pm 10,9$ vs $21 \pm 7 \mathrm{U} / \mathrm{L}$, respectivamente). La lactato deshidrogenasa/LDH y las plaquetas también mostraron diferencias significativas entre ambos grupos, aunque de todos los parámetros de laboratorio evaluados, las enzimas hepáticas AST y ALT fueron las que mostraron mayor diferencia al ingreso y durante la evolución en las pacientes que desarrollaron síndrome de HELLP.

Evaluando el desempeño de los parámetros de laboratorio al ingreso de las pacientes con síndrome de HELLP (Figura 1), AST y ALT tienen un área bajo la curva ROC de 0,828 (IC95\% 0,770-0,885) y 0,813 (IC95\% 0,745-0,881), respectivamente, observándose que son las primeras pruebas bioquímicas en alterarse (Tabla II).

\section{Tabla II}

\section{PUNTOS DE CORTE DE LOS PARÁMETROS DE LABORATORIO}

\begin{tabular}{lccc}
\hline Laboratorio & Punto de corte & VPP (\%) & VPN (\%) \\
\hline DHL (U/L) & 611 & 59,0 & 78,6 \\
AST (U/L) & 34,5 & 67,5 & 75,7 \\
ALT (U/L) & 28,5 & 72,6 & 85,4 \\
Plaquetas & 231.500 & 18,8 & 55,1 \\
(x mm3) & & & \\
\hline
\end{tabular}

VPP: Valor predictivo positivo. VPN: Valor predictivo negativo.

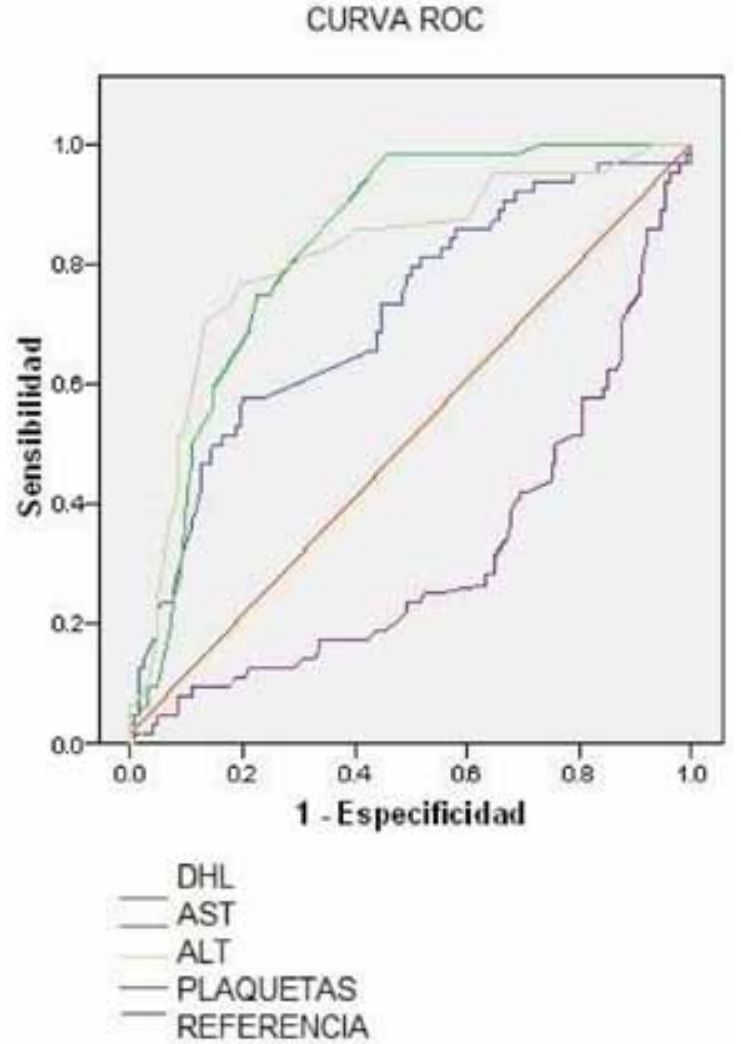

Figura 1. Curva ROC de los parámetros de laboratorio de DHL, plaquetas, AST, ALT, para determinar presencia de HELLP al ingreso de las pacientes con preclampsia severa. 
De las cuatro pruebas de laboratorio, la ALT es la que mejor predice el comportamiento de la PES cuando evoluciona a síndrome de HELLP, con un VPP de $72,6 \%$. Al comparar las características clínicas se observó que la epigastralgia durante la evolución de las pacientes con PES que desarrollaron síndrome de HELLP presenta una LR+ alta $(18,4 ;$ IC95\% 7,69-44,6) y un alto VPP $(90,2 \%$; IC95\% 81,05-99,34), lo que significa que la presencia de epigastralgia es un síntoma que sugiere tempranamente el desarrollo del síndrome de HELLP en las pacientes con diagnóstico de PES. La cefalea, las náuseas y el vómito no mostraron ser síntomas que sugieran el desarrollo temprano del síndrome de HELLP.

Utilizando los puntos de corte de las transaminasas y la epigastralgia, los cuales fueron los parámetros que mostraron mejor desempeño como prueba predictora de síndrome de HELLP en PES, se combinaron los tres criterios (AST, ALT y epigastralgia) para mejorar la probabilidad diagnóstica antes de haber cumplido todos los criterios del síndrome de HELLP. La combinación de estos 3 criterios, aplicada a las pacientes con preeclampsia severa mejora la probabilidad diagnóstica del síndrome de HELLP, presentando un VPP del 95,5\% y un LR+ de 42,0 .

\section{DISCUSIÓN}

El síndrome de HELLP es considerado como una de las complicaciones de la PES, comúnmente el diagnóstico se basa en criterios de laboratorio, pero uno de los mayores problemas con la detección temprana radica en su presentación clínica inespecífica, en donde los signos y síntomas se pueden encontrar en pacientes con PES sin síndrome de HELLP $(9,10)$.

Weinstein (11), reporta una sensibilidad de $86 \%$ de dolor epigástrico en pacientes con síndrome de HELLP, mientras que Sibai cols (12), informan una sensibilidad de $66 \%$ para este síntoma. En nuestro estudio el dolor en epigastrio fue la manifestación clínica que reportó mejor sensibilidad $(71,8 \%)$ y VPP $(90,2 \%)$.

En el estudio realizado por Martin y cols (13), se midieron los parámetros de laboratorio al ingreso de pacientes con PES con el fin de buscar un predictor de morbilidad materna, encontrando que en estas los principales predictores son la elevación de LDH, transaminasas y ácido úrico. En el estudio de Carpani y cols (14), sobre evaluación de los parámetros maternos diagnósticos como predictor de severidad de síndrome de HELLP al ingreso, las transaminasas y la LDH son los parámetros con mayor peso. Lo anterior se relaciona con el modelo de PIERS (Preeclampsia integrated estimate of risk) el cual es un predictor de resultado adverso en las madres con preeclampsia e incluye dentro de sus parámetros la AST (15).
Tanto el dolor en epigastrio como el aumento de las transaminasas nos hablan de una alteración a nivel hepático. Es bien conocido que el hígado es un órgano comprometido en el síndrome de HELLP, donde los depósitos de fibrina como consecuencia de la hemólisis obstruyen los sinusoides causando daño hepatocelular y hemorragia periportal, que traen como consecuencia la elevación de las pruebas de función hepática y el dolor en epigastrico (16). Lo anterior nos lleva a definir que a las pacientes con PES que presenten un aumento en las transaminasas y epigastralgia durante su evolución, se les debe realizar una vigilancia más estrecha y una aproximación diagnóstica más agresiva con el objetivo de identificar tempranamente el síndrome de HELLP, y así disminuir la mortalidad materna y perinatal que se relacionan con esta afección.

Por último, en nuestro estudio, las pacientes que desarrollaron síndrome de HELLP presentaron una edad gestacional más temprana que las pacientes con PES que no desarrollaron este síndrome (31,9 vs 36,2 semanas). Existen otros estudios $(17,18)$ donde reportan que la edad gestacional es un importante predictor de complicaciones maternas en la preclampsia.

\section{CONCLUSIÓN}

En pacientes con diagnóstico de PES, la presencia de epigastralgia y elevación de las transaminasas son fuertes predictores de desarrollo de síndrome de HELLP, por tal razón en este grupo de pacientes se debe controlar activamente la alteración de estos parámetros.

\section{REFERENCIAS}

1. Haram K, Svendsen E, Abildgaard U. The HELLP syndrome: Clinical issues and management. A review. BMC Pregnancy Childbirth 2009;9:8-23.

2. Magee LA, Helewa M, Moutquin JM, von Dadelszen $P$. Diagnosis, evaluation, and management of the hypertensive disorders of pregnancy. J Obstet Gynaecol Can 2008;30(3 suppl):S1-48.

3. Steegers EA, von Dadelszen P, Duvekot JJ, Pijnenborg R. Pre-eclampsia. Lancet 2010;376(9741):631-44.

4. Joshi D, James A, Quaglia A, Westbrook RH, Heneghan MA. Liver disease in pregnancy. Lancet 2010;375(9741):594-605.

5. Guzel AL, Kuyumcuoglu U, Celik Y. Are maternal and fetal parameters related to perinatal mortality in HELLP syndrome? Arch Gynecol Obstet 2011;283:1227-32.

6. Connealy BD, Carreno CA, Kase BA, Hart LA, Blackwell SC, Sibai BM. A history of prior preeclampsia as a risk factor for preterm birth. Am J Perinatol 2013; [Epub ahead of print].

7. Martin JN Jr, Blake PG, Lowry SL, Perry KG Jr, Files JC, Morrison JC. Pregnancy complicated by preeclampsia-eclampsia with the syndrome of hemolysis, elevated liver enzymes and low platelet count: 
how rapid is postpartum recovery? Obstet Gynecol 1990;76:737-41.

8. Martin JN Jr, Blake PG, Perry KG, McCaul JF, Hess LW, Martin RW. The natural history of HELLP syndrome: patterns of disease progression and regression. Am J Obstet Gynecol 1991;164:1500-13.

9. Isler CM, Martin JN Jr. Preeclampsia: pathophysiology and practice considerations for the consulting nephrologist. Semin Nephrol 2002;22:54-64

10. Vigil-De Gracia P, Ortega-Paz L. Pre-eclampsia/ eclampsia and hepatic rupture. Int J Gynecol Obstet 2012;118:186-9.

11. Weinstein L. Preeclampsia/eclampsia with hemolysis, elevated liver enzymes and thrombocytopenia. Obstet Gynecol 1985;66:657-60.

12. Sibai BM. Diagnosis, Controversies, and Management of the Syndrome of hemolysis, elevated liver enzymes, and low platelets count. Obstet Gynacol 2004;103:981-92.

13. Martin JN Jr, May WL, Magann EF, Terrone DA, Rinehart BK, Blake PG. Early risk assessment of severe preeclampsia: admission battery of symptoms and laboratory tests to predict likelihood of subsequent significant maternal morbidity. Am J Obstet Gynecol 1999;180:1407-14.

14. Carpani G, Bozzo M, Ferrazzi E, D'Amato B, Pizzotti D, Radaelli T, Moroni G, Pardi G. The evaluation of maternal parameters at diagnosis may predict $\mathrm{HE}$ LLP syndrome severity. J Matern Fetal Neonatal Med 2003;13:147-51.

15. Von Dadelszen P, Payne B, Li J, Ansermino JM, Broughton Pipkin F, Cote AM, et al; PIERS Study Group. Prediction of adverse maternal outcomes in pre-eclampsia: development and validation of the fullPIERS model. Lancet 2011;377(9761):219-27.

16. Baxter JK, Weinstein L. HELLP syndrome: The state of art. Obstet Gynecol Surv 2004;59:838-45.

17. Nisell H, Palm K, Wolff K. Prediction of maternal and fetal complications in preeclampsia. Acta Obstet Gynecol Scand 2000;79:19-23.

18. Ganzevoort W, Rep A, de Vries J, Bonsel GJ, Wolf $\mathrm{H}$. Prediction of maternal complications and adverse infant outcome at admission for temporizing management of early-onset severe hypertensive disorders of pregnancy. Am J Obstet Gynecol 2006;195:495-503. 